\title{
Difference-quotient turbulence model: A generalization of Prandtl's mixing-length theory
}

\author{
Peter W. Egolf \\ Swiss Federal Laboratories for Materials Testing and Research, CH-8600 Dübendorf, Switzerland
}

(Received 21 September 1993)

\begin{abstract}
A difference mean-velocity turbulence model to calculate quasistationary fully developed turbulent motion is presented. It takes account of the various eddy sizes in turbulent flows. As a consequence, some gaps between semiempirical closure models and sophisticated theories, describing eddy energy cascades, are reduced. In a first-order expansion, the model is identical to Prandtl's mixing-length theory, which is still contained in modern numerical computation schemes, e.g., $k-\epsilon$ models.
\end{abstract}

PACS number(s): 47.27.Vf, 47.27.Qb

\section{INTRODUCTION}

Scientists have investigated turbulent phenomena for hundreds of years. Leonardo da Vinci (1452-1519), an artist, architect, and natural scientist, studied turbulent flows and produced several drawings showing eddies of various size. Based on such observations, today we have knowledge of energy-cascade models describing the turbulent kinetic energy of flow as a function of eddy size or of the related wave number (e.g., Kolmogorov-Oboukov law). In these theories it is assumed that the forcing of a system generates large eddies, which decay and become smaller and smaller until viscous dissipation sets in, which is dependent on the viscous stress of the fluid flow (rate of deformation tensor). Thus turbulent kinetic energy is transferred from small-scale eddies into molecular motion. In some recent approaches turbulent domains are thought to be separated by fractal boundaries from laminar-flow regions with potential flow. This phenomenon is named spatial intermittency. Despite such successful theoretical explanations many mathematical descriptions of fully developed turbulent flows are still not complete, e.g., statistical kinetic theories of phenomena with memory effects and long-range correlations (strange kinetics). This deficiency is a hindrance for further success in many research topics of other activity fields, e.g., dynamic meteorology, oceanography, chemistry, and astronomy. [1].

In the early days of research on turbulence, crude models were proposed to describe momentum transport or the spreading out of other transferable quantities in turbulent flows, such as heat, contaminants, pollutants, etc. A1though these first attempts are not taken very seriously by many dedicated analysts - they are known as empirical models - still, some of these simple closures were of great help to engineers, who had to deal with daily-life flow problems. Studying the old models extensively, some can immediately be sorted out because of missing symmetry or invariance properties. On the other hand, three semi-empirical approaches, advanced by Prandtl, seem to be very fruitful and worthy of further examination.

In this article, an alternative model of turbulence is proposed, originating from studies of fully developed tur- bulent wake flows. For this kind of free shear flow, by mass- and momentum-flux considerations, one can analytically calculate the tangential Reynolds shear stress [2]. An accurate model of turbulence must also yield the same result for this particular flow problem. The alternative model fulfills this requirement.

Not long ago, it was realized that this alternative model is closely related to the ideas of Prandtl on turbulent momentum transport. Moreover, the discovery of a certain consistency of some of these theories could be a hint that they contain more correct information on the underlying mechanisms of fully developed turbulence than we have assumed up to the present. Therefore, the aim of this paper is to outline features which the different models have in common. It will even be shown that Prandtl's most famous approach - the mixing-length theory-is exactly the first-order equation of the proposed model.

\section{THREE MODELS OF PRANDTL}

It is advantageous to make a statistical approach to turbulent phenomena and to split quantities such as

$$
\chi=\bar{\chi}+\chi^{\prime}, \quad \chi \in\left\{u_{1}, u_{2}, u_{3}, \ldots\right\}
$$

into a time-averaged value $\bar{\chi}$ and the corresponding fluctuation $\chi^{\prime}$. In Eq. (1) we have introduced the three velocity components. The mean values are determined by finite averaging times that are long compared with the time scales of the turbulent flow but small in comparison with transient times produced by changes of the excitation of the system. In the quasistationary flows we are going to study, there is no such transient behavior, and mainly because of this, the mean values do not depend on time any further. Splitting up all the quantities-as shown in Eq. (1) - in the continuity equation and the Navier-Stokes equation, and afterwards taking the average of all their terms, one ends up with the equations for the mean values. These equations contain correlation products of the velocity fluctuations, e.g., $\overline{u_{2}^{\prime} u_{1}^{\prime}}$. At the core of the (older phenomenological) models on turbulence is the intrinsic believe that such second-order correlations, e.g., tangential Reynolds stresses

$$
\tau_{k l}(x)=-\rho \overline{u_{k}^{\prime} u_{l}^{\prime}}(x)
$$


are determined by the mean velocity field $\bar{u}=\left(\bar{u}_{1}, \bar{u}_{2}, \bar{u}_{3}\right)$. Following Speziale [3], we then assume that the Reynolds stresses are functionals $F_{k l}$ of the mean velocity field at all points $x^{*}$ :

$$
\tau_{k l}(x)=F_{k l}\left(\bar{u}\left(x^{*}\right), x\right), \quad x^{*} \in V,
$$

where $V$ is a bounded or unbounded volume of the fluid, containing and depending on the point $x=\left(x_{1}, x_{2}, x_{3}\right)$. It is important to note that if there exists an $x^{*} \neq x$ in $V$, this approach includes nonlocal effects. Furthermore, an obvious advantage of restricting ourselves to mean quasistationary flows is that in cases with nonlocalities no violation of special relativity will occur.

At the basis of many models on turbulence is an assumption made by Boussinesq [4]. By a comparison of turbulence stresses with stresses in laminar flow caused by viscosity, he proposed that the Reynolds stresses are also proportional to the (mean) velocity gradient:

$$
\tau_{k l}(x)=\epsilon \frac{\partial \overline{u_{l}}}{\partial x_{k}} .
$$

An important difference is that the eddy viscosity $\epsilon$ is not a material constant like the kinematic viscosity $v$; it therefore depends on the fluid motion. We will always assume the main flow direction to be in the $x_{1}$ direction. The existence of such a main flow direction in free turbulent flows implies some significant simplifications in the equations of motion, in Boussinesq's assumption (4), and the turbulence model under consideration [2]. In the past 20 years several authors [5-7] have criticized the phenomenological gradient approaches.

Criticism 1. One-point models, such as gradient laws, are found to be inherently unsuitable to describe turbulent momentum transport.

Some of these authors have pointed out that the analogy between molecular and turbulent transport is questionable. In the theory of kinetic gases the size of the molecules is assumed to be small compared with the mean free path. In contrast, the size of the largest interacting eddies, i.e., the "mixing length," is not even small compared with the characteristic length scale of the flow under consideration.

In kinetic theory of gases, the viscosity is proportional to the product of the root-mean-square velocity and the mean free path of the molecules. In analogy, Prandt [8]-in his mixing-length theory, based on (4) introduced the mean velocity by a first-order expansion with a turbulence length scale, the mixing length $l$ :

$$
\tau_{21}\left(x_{1}, x_{2}\right)=\rho l^{2}\left|\frac{\partial \overline{u_{1}}}{\partial x_{2}}\right| \frac{\partial \overline{u_{1}}}{\partial x_{2}} .
$$

The absolute value of the first gradient is necessary to obtain the correct sign of the shear stress. For an extensive discussion of Prandtl's physical ideas consult Ref. [9]. This mixing-length theory was the first reasonable turbulence model and was applied with great success in free shear flows and turbulent wall flows. It also found appreciation in other scientific disciplines, e.g., in dynamical meteorology for the description of planetary boundary layers [10]. Nevertheless, it has two shortcomings, the first of which follows.

Criticism 2. Comparing measured and calculated mean velocity profiles $\overline{u_{1}}$ in function of $x_{2}$, one finds deviations at the points of vanishing derivative.

In 1942 Prandtl [11], knowing about this inadequacy, proposed an extended model containing in the eddy viscosity a mean gradient over a distance $l^{\prime}$ :

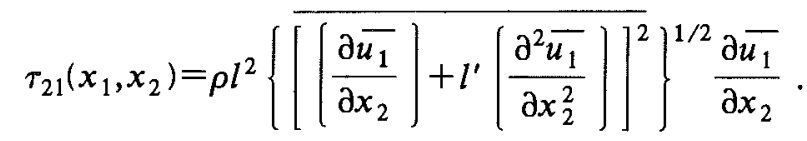

He then assumed that the second mixing length $l^{\prime}$ is in the positive and negative directions statistically equally distributed, so that in this mean-gradient theory, on an average the cross terms cancel out:

$\tau_{21}\left(x_{1}, x_{2}\right)=\rho l^{2}\left[\left(\frac{\partial \overline{u_{1}}}{\partial x_{2}}\right)^{2}+l^{2}\left[\frac{\partial^{2} \overline{u_{1}}}{\partial x_{2}^{2}}\right]^{2}\right]^{1 / 2} \frac{\partial \overline{u_{1}}}{\partial x_{2}}$.

In Eq. (7) we have written $l^{\prime 2}$ instead of $l^{\prime 2}$. With this model the calculations became more accurate, but also considerably more complicated. Furthermore, a second shortcoming of the mixing-length theory was not avoided by the previous extension, leading to Eq. (7).

Criticism 3. Calculating the mixing lengths with these models and experimental data leads to characteristic length scales which are much smaller than the largest eddies observed in the flow under consideration.

Therefore, Prandt1 [11], in a third approach - the free shear-layer theory - tried to relate the eddy viscosity to the overall flow conditions, namely, the width $b$ of the turbulent zone and the greatest mean velocity difference:

$$
\tau_{21}\left(x_{1}, x_{2}\right)=\kappa \rho b\left(\overline{u_{1_{\max }}}-\overline{u_{1_{\min }}}\right) \frac{\partial \overline{u_{1}}}{\partial x_{2}} .
$$

$\kappa$ simply denotes a constant. Görtler [12] calculated velocity functions with this approach, which he found to be in good agreement with experimental results, but still with one exception.

Criticism 4. At the boundary of the mixing zone there are deviations, because the eddy viscosity does not vanish there.

We can rewrite Eq. (8) in the following way:

$$
\tau_{21}\left(x_{1}, x_{2}\right)=\kappa \rho b^{2} \frac{\partial \overline{u_{1}}}{\partial x_{2}}\left(\frac{\overline{u_{1_{\max }}}-\overline{u_{1_{\min }}}}{b}\right) \text {. }
$$

The velocities $\overline{u_{1_{\max }}}$ and $\overline{u_{1_{\min }}}$ are usually separated exactly by the distance $b$. Therefore, this was probably the first and most simple nonlocal model, with a very stiff difference quotient, which does not even depend on $x_{2}$. As we will see in Sec. III, it would be much more convenient to eliminate the index max in this equation. A Taylor expansion of the mean gradient in large parentheses, in first order, would then just yield Eq. (5). Omitting the constant, instead of the mixing length $l$, in this case, the entire width $b$ of the turbulent domain appears. If the local length 


$$
L=\frac{\frac{\partial \overline{u_{1}}}{\partial x_{2}}}{\frac{\partial^{2} \overline{u_{1}}}{\partial x_{2}^{2}}},
$$

introduced by von Karman [13], is at least one order greater than the second mixing length $l^{\prime}$,

$$
|L| \gg\left|l^{\prime}\right|,
$$

then a Taylor expansion of the slightly altered Eq. (9) up to second order would exactly equal Prandtl's second model (7).

The extended analysis of Prandtl's theories shows how closely related his different approaches actually are: one being only a slightly corrected or generalized version of the other. After rewriting the free shear-layer model (8), we have obtained a very simple difference-quotient theory. It appears as a preliminary stage of the model, which will be presented in the remainder of this article.

\section{THE DIFFERENCE-QUOTIENT MODEL}

As the name indicates, the alternative differencequotient model contains a difference quotient instead of the usually applied first-derivative law [14]

$\tau_{2,1}=-\rho \overline{u_{2}^{\prime} u_{1}^{\prime}}=\rho \chi_{2} \frac{d b}{d x_{1}}\left(\overline{u_{1}}-\overline{u_{1_{\min }}}\right) \frac{\overline{u_{1_{\max }}}-\overline{u_{1}}}{x_{2_{\max }}-x_{2}}$.

$\chi_{2} \in\left\{x_{2}, b\right\}$ is a variable or characteristic length scale of the flow, perpendicular to the main flow direction and always can be determined by symmetry arguments. In certain flow problems the variables with an index min or max must be replaced by the infimum (supremum) of the variable or function under consideration. Note that this rather unusual model does not make use of the phenomenological eddy viscosity concept (4).

The difference quotient, which for certain locations is a mean gradient over a very large domain, introduces a nonlocality. For that reason criticism 1 does not apply to this model. Taking this approach into account, several mean properties of different turbulent shear flows have been calculated and in every case analytical solutions could be obtained. They show no deviations from measurements at the points of vanishing derivative (criticism 2). The shortcoming (criticism 3) doesn't apply either because $\chi_{2}$ is a large length scale, e.g., the width $b$ of the entire turbulent zone. If we also denote the expression in front of the difference quotient as "eddy viscosity," we recognize that it vanishes at the boundary of the turbulent zone, where $\overline{u_{1}} \rightarrow \overline{u_{1_{\min }}}$ (criticism 4). Note that the correction $\left(\overline{u_{1}}-\overline{u_{1_{\min }}}\right)$ instead of $\left(\overline{u_{1_{\max }}}-\overline{u_{1_{\min }}}\right)$ in the eddy viscosity requires the second velocity difference $\left(\overline{u_{1} \max }-\overline{u_{1}}\right)$ in Eq. (12) because of symmetry arguments, e.g., exchanging $x_{2}$ and $-x_{2}(\min \leftrightarrow \max )$ in a coflow [15]. In addition to velocity differences, it also is convenient to introduce differences in the coordinates. From this discussion we conclude that the difference-quotient model is a natural continuation of Prandtl's ideas on momentum transfer. All shortcomings of the older theories discussed no longer apply to the differencequotient model.

Direct numerical simulations of turbulent flows of Bernard and Handler [7] producing several ensembles of fluid particle paths have shown that the displacement transport mechanism has a natural representation in terms of a global distribution of the mean velocity gradient, as it occurs in this model. These authors further believe that their result could be of some advantage in the design of improved Reynolds stress models. Therefore, the difference-quotient turbulence model, being completely in correspondence with these recent numerical results, may combine new and old ideas on turbulence.

\section{A GENERALIZATION OF THE MIXING-LENGTH THEORY}

We now expand $\overline{u_{1_{\min }}}\left(x_{1}\right)$ and $\overline{u_{1_{\max }}}\left(x_{1}\right)$ at $x_{2}$ in the $x_{2}$ direction in a Taylor series. Inserting these expansions into (12) yields

$$
\begin{gathered}
\tau_{21}=\rho \frac{d b}{d x_{1}} \chi_{2} \sum_{m, n=1}^{\infty} \frac{(-1)}{m ! n !}\left(x_{2 \min }-x_{2}\right)^{m}\left(x_{2_{\max }}-x_{2}\right)^{n-1} \\
\times\left[\frac{\partial^{m} \overline{u_{1}}}{\partial x_{2}^{m}}\right]\left[\frac{\partial^{n} \overline{u_{1}}}{\partial x_{2}^{n}}\right]
\end{gathered}
$$

Formally, the nonlocality has been transformed into an infinite dimensionality. The terms of order zero have canceled out, and to first order this infinite order equation reduces to Prandtl's mixing-length theory:

$$
\tau_{21}=\rho l^{2}\left(\frac{\partial \overline{u_{1}}}{\partial x_{2}}\right)\left(\frac{\partial \overline{u_{1}}}{\partial x_{2}}\right),
$$

with the following square product of the mixing length:

$$
l^{2}=l_{1} l_{2}=\beta \chi_{2}\left(x_{2}-x_{2_{\text {min }}}\right), \beta=\frac{d b}{d x_{1}},
$$

where we have preferred to distinguish the mixing length $l_{1}$ in the $x_{1}$ direction from $l_{2}$ in the $x_{2}$ direction. An extensive study of different flows shows that in some cases, a power-law expansion as given in (13) is not very appropriate.

From experimental investigations, Prandtl found corrections necessary which correspond to higher-order terms in the mean velocity. However, he never gave up the empirical Boussinesq approach. He therefore always included his higher-order contributions into the eddy viscosity. Actually, in the older models, it was customary to adapt calculations to experimental results by choosing some suitable eddy viscosity. However, the only small inconsistency in Prandtl's theories is the index max in his free shear-layer model (8). Apart from this, all the presented models are fully compatible with each other. The difference-quotient model is the theory of highest order, a generalization that leads to the most complete description of turbulent behavior of all the models, discussed in Secs. II and III. Higher-order terms do not occur anymore in a tangled way; they now succeed each other with increasing order as it is common in series expansions. 


\section{THE PERFORMANCE OF THE MODEL}

\section{A. Introduction}

For a plane wake flow (Fig. 1), one can calculate the Reynolds shear stress from mass and momentum considerations [2]. An accurate turbulence model has to lead to the same result, a requirement which, to the author's best knowledge, is not fulfilled by other turbulence models. In Sec. V B it will be shown that with the differencequotient model the desired solution can be produced. Then, for a turbulent round jet in a quiescent surrounding $\left(U_{G}=0\right)$ and in a basic surrounding flow, with a velocity $U_{G}$ at least one order greater than the maximal mean excess velocity (Fig. 2), several important mean flow properties are analytically calculated. These results are compared with measurements as far as experimental data were available.

\section{B. The turbulent plane wake flow}

A cylinder with diameter $d$ is mounted into an uniform potential flow with a velocity $U_{G}$. The mean velocity of the perturbed flow is

$$
\overline{u_{1}}=U_{G}-\overline{u_{1}^{*}} \text {. }
$$

The Reynolds number of the flow shall be

$$
\operatorname{Re}=\frac{U_{G} d}{v}>800
$$

to guarantee a fully turbulent disturbance with a mean velocity $\bar{u}_{1}^{*}$ pointing upstream in the $-x_{1}$ direction (Fig. 1). The quantity $v$ still denotes the kinematic viscosity.

At locations

$$
\xi_{1}=\frac{x_{1}-p}{d}>90
$$

a self-similar flow regime-with Reynolds-number similarity-occurs. It has its origin at $x_{1}=p$ some distance upstream from the axis of the cylinder. Therefore, in the following subsections use can be made of several similarity relations:

$$
\begin{aligned}
& {\overline{u_{1}}}^{*}=U_{G}\left(\frac{x_{1}-p}{k d}\right)^{p_{1}} f_{1}(\eta), \\
& {\overline{u_{2}}}^{*}=U_{G}\left(\frac{x_{1}-p}{k d}\right)^{p_{2}} f_{2}(\eta),
\end{aligned}
$$

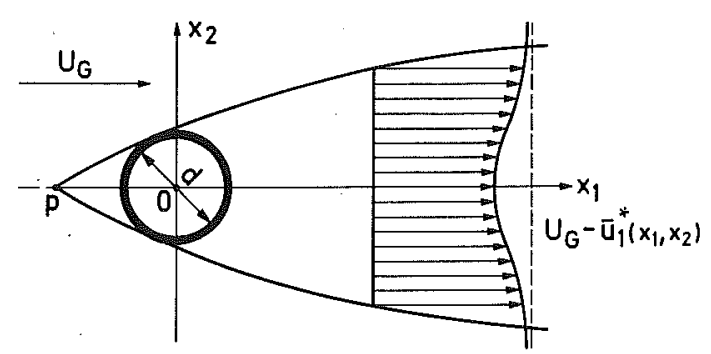

FIG. 1. Turbulent plane wake flow behind a cylinder.

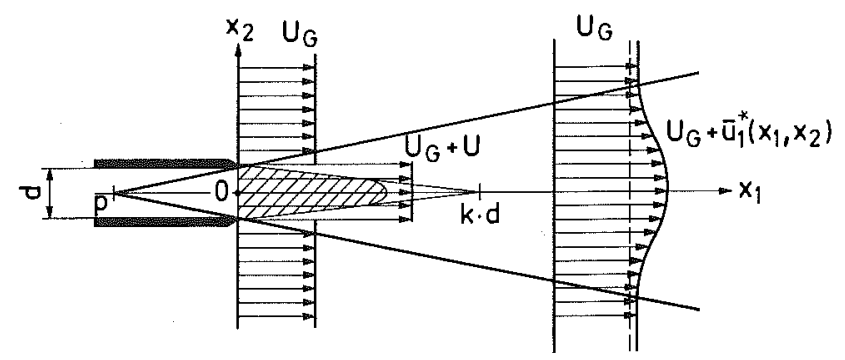

FIG. 2. Turbulent round jet in a surrounding flow with velocity $U_{G}$.

$$
\begin{aligned}
& \overline{u_{2}^{\prime} u_{1}^{\prime}}{ }^{*}=-U_{G}^{2}\left(\frac{x_{1}-p}{k d}\right)^{p_{2,1}} f_{2,1}(\eta), \\
& \eta=\frac{x_{2}}{b}, \quad b=\beta\left(\frac{x_{1}-p}{k d}\right)^{p_{0}} k d .
\end{aligned}
$$

The quantity $b$ denotes a characteristic width of the turbulent free shear flow and $k$ is a constant. Now we can apply the following operations:

$$
\begin{aligned}
& \frac{\partial}{\partial x_{1}} \rightarrow-p_{0} \frac{1}{x_{1}-p} \eta \frac{d}{d \eta}, \\
& \frac{\partial}{\partial x_{2}} \rightarrow \frac{1}{\beta k d}\left(\frac{k d}{x_{1}-p}\right)^{p_{0}} \frac{d}{d \eta} .
\end{aligned}
$$

The continuity equation and Euler equation in Cartesian coordinates, describing the time-averaged behavior of a turbulent plane wake - after applying a scale analysis are [2]

$$
\begin{aligned}
& \frac{\partial}{\partial x_{1}}\left(\frac{\overline{u_{1}^{*}}}{U_{G}}\right)+\frac{\partial}{\partial x_{2}}\left[\frac{u_{2}^{*}}{U_{G}}\right)=0, \\
& \frac{\partial}{\partial x_{1}}\left(\frac{u_{1}^{*}}{U_{G}}\right)-\frac{\partial}{\partial x_{2}}\left(\frac{\overline{u_{2}^{\prime} u_{1}^{\prime}}}{U_{G}^{2}}\right)=0 .
\end{aligned}
$$

Substituting (19) - (24) into (25) and (26), and requiring ordinary differential equations containing only the variable $\eta$, we obtain

$$
\begin{aligned}
& p_{0}+p_{1}-p_{2}=1, \\
& p_{1} f_{1}-p_{0} \eta \frac{d f_{1}}{d \eta}+\frac{1}{\beta} \frac{d f_{2}}{d \eta}=0, \\
& p_{0}+p_{1}-p_{2,1}=1, \\
& p_{1} f_{1}-p_{0} \eta \frac{d f_{1}}{d \eta}+\frac{1}{\beta} \frac{d f_{2,1}}{d \eta}=0 .
\end{aligned}
$$

To determine the exponents of all the power laws, two further equations have to be known. The first one follows from the requirement that the Reynolds shear stress scaled with the square of the mean velocity in the main flow direction is independent of $x_{1}$ :

$$
2 p_{1}-p_{2,1}=0 \text {. }
$$


From (26), by an integration vertical to the main flow direction, we derive

$$
\int_{-\infty}^{\infty} \frac{\overline{u_{1}^{*}}}{U_{G}} d x_{2}=\text { const }
$$

because the Reynolds shear stress vanishes at infinity $( \pm \infty)$. Important to obtain self-similarity is the assumption that the mean disturbance is at least one order of magnitude smaller than the undisturbed mean flow velocity:

$$
\frac{{\overline{u_{1}}}^{*}}{U_{G}} \ll 1
$$

Studying momentum fluxes through a closed rectangular boundary far from the cylinder, with assumption (33), we obtain

$$
\int_{-\infty}^{\infty} \rho\left[U_{G}^{2}-\left(U_{G}-\overline{u_{1}^{*}}\right)^{2}\right] d x_{2}=2 \rho U_{G} \int_{-\infty}^{\infty} \overline{u_{1}}{ }^{*} d x_{2} \text {. }
$$

The mass flux entering through the boundary from the left-hand side is greater than the outflowing flux leaving the bounded domain on the right. Therefore, the following momentum loss:

$$
-\rho U_{G} \int_{-\infty}^{\infty} \overline{u_{1}^{*}} d x_{2}
$$

results. After Newton's second axiom the sum of (34) and (35) is equal to the mean force (drag) acting on the cylinder:

$$
\frac{\bar{F}}{\rho U_{G}^{2} d}=\frac{1}{d} \int_{-\infty}^{\infty} \frac{\overline{u_{1}^{*}}}{U_{G}} d x_{2},
$$

an equation that leads to the fourth relation

$$
p_{0}+p_{1}=0 \text {. }
$$

The system of equations (27), (29), (31), and (37) has the following results:

$$
p_{0}=\frac{1}{2}, p_{1}=-\frac{1}{2}, p_{2}=-1, p_{2,1}=-1 \text {. }
$$

The width of the turbulent domain increases with a root dependence. With these results, after an integration of Eq. (30), the scaled Reynolds shear stress is obtained:

$$
f_{2,1}=\frac{\beta}{2}\left[\int_{0}^{\eta}\left[f_{1}(\xi)+\xi \frac{d f_{1}(\xi)}{d \xi}\right] d \xi\right] \text {. }
$$

An integration by parts yields

$$
f_{2,1}=\frac{\beta}{2} \eta f_{1} \text {. }
$$

Finally, we have derived an analytical solution of the Reynolds shear stress:

$$
\frac{\overline{u_{2}^{\prime} u_{1}^{\prime}}}{U_{G}^{2}}=-\frac{1}{2}\left(\frac{x_{2}}{x_{1}-p}\right) \frac{{\overline{u_{1}}}^{*}}{U_{G}} .
$$

It must be possible to derive this result with an accurate turbulence model.

Now, the difference-quotient model can directly be applied to the plane wake flow:

$$
\begin{aligned}
\overline{u_{2}^{\prime} u_{1}^{\prime}}=- & x_{2} \frac{d b}{d x_{1}}\left[U_{G}-\overline{u_{1}^{*}}\left(x_{1}, x_{2}\right)\right] \\
& \times \frac{U_{G}-\left[U_{G}-\overline{u_{1}}{ }^{*}\left(x_{1}, x_{2}\right)\right]}{b-x_{2}} .
\end{aligned}
$$

This follows because

$$
\chi_{2}=x_{2}, \overline{u_{1_{\min }}}=0, \overline{u_{1_{\max }}}=U_{G} .
$$

The maximal mean velocity $U_{G}$ occurs at infinity: $x_{2_{\max }} \rightarrow \infty$ (Fig. 1). Therefore, it is suitable to set $x_{2_{\max }}=b$-as we already have done in Eq. (42) — with the great advantage to obtain a Reynolds shear stress that does not depend on the definition of the width $b$ :

$$
\overline{u_{2}^{\prime} u_{1}^{\prime}}=\lim _{b \rightarrow \infty}\left\{-x_{2} \frac{1}{b} \frac{d b}{d x_{1}} U_{G} \frac{\overline{u_{1}^{*}\left(x_{1}, x_{2}\right)}}{1-\left[\frac{x_{2}}{b}\right]}\right\} \text {. }
$$

Because the scaling behavior of the width of the wake, given in Eq. (22), also has to be valid in the limit when $b$ tends to infinity, a natural consequence is $b \rightarrow \infty \Longleftrightarrow \beta \rightarrow \infty$. This assumption leads to the following result:

$\frac{\overline{u_{2}^{\prime} u_{1}^{\prime *}}}{U_{G}^{2}}=-\frac{x_{2}}{b} \frac{d b}{d x_{1}}\left(\frac{\overline{u_{1}^{*}}}{U_{G}}\right)=-\frac{1}{2}\left(\frac{x_{2}}{x_{1}-p}\right) \frac{\overline{u_{1}^{*}}}{U_{G}}$,

which is identical to the analytically derived result (41).

To fulfill (41) with a model containing Boussinesq's approach only, one would have to deal with

$$
f_{1} \propto \frac{d f_{1}}{d \eta}=f_{1} \propto \exp (\eta),
$$

a rather unsuitable function for a mean velocity profile.

For a plane wake the Reynolds shear stress had to be obtained by a delicate limiting procedure, because in this case the difference quotient vanishes. The eddy viscosity of our model, namely, the physical properties in front of the difference quotient, have to tend to infinity to give a bounded Reynolds shear stress. Therefore, from this example we learn that the entire relation forms an indivisible unit.

\section{The turbulent round jet in a quiescent surrounding}

Similar to Sec. VB, the basic flow equations in cylindrical coordinates, describing the time-averaged behavior of a turbulent round jet, are

$\frac{\partial \bar{u}_{1}^{*}}{\partial x_{1}}+\frac{1}{x_{2}} \frac{\partial}{\partial x_{2}}\left(x_{2}{\overline{u_{2}}}^{*}\right)=0$,

${\overline{u_{1}}}^{*} \frac{\partial \overline{u_{1}}}{\partial x_{1}}+\overline{u_{2}} \frac{\partial \overline{u_{1}^{*}}}{\partial x_{2}}+\frac{1}{x_{2}} \frac{\partial}{\partial x_{2}}\left(x_{2} \overline{u_{2}^{\prime} u_{1}^{\prime}}\right)=0$,

where again the properties have been split up into a basic flow and a perturbation:

$$
\overline{u_{1}}=U_{G}+\overline{u_{1}^{*}} \text {. }
$$


We assume the following similarity relations:

$$
\begin{aligned}
& {\overline{u_{1}}}^{*}=U\left(\frac{x_{1}-p}{k d}\right)^{p_{1}} f_{1}(\eta), \\
& \overline{u_{2}^{*}}=U\left(\frac{x_{1}-p}{k d}\right)^{p_{2}} f_{2}(\eta), \\
& \overline{u_{2}^{\prime} u_{1}^{*}}=-U^{2}\left(\frac{x_{1}-p}{k d}\right)^{p_{2,1}} f_{2,1}(\eta), \\
& \eta=\frac{x_{2}}{b}, \quad b=\beta\left(\frac{x_{1}-p}{k d}\right)^{p_{0}} k d .
\end{aligned}
$$

Substituting these self-similar functions into the continuity equation yields

$$
\begin{aligned}
& p_{0}+p_{1}-p_{2}=1, \\
& p_{1} f_{1}-p_{0} \eta \frac{d f_{1}}{d \eta}+\frac{1}{\beta} \frac{1}{\eta} \frac{d}{d \eta}\left(\eta f_{2}\right)=0 .
\end{aligned}
$$

The Euler equation becomes

$$
\begin{aligned}
\frac{1}{k d}\{ & {\left[\left(\frac{U_{G}}{U}\right]+\left[\frac{x_{1}-p}{k d}\right]^{p_{1}} f_{1}\right]\left[\frac{x_{1}-p}{k d}\right]^{p_{1}-1} } \\
\times & {\left[p_{1} f_{1}-p_{0} \eta \frac{d f_{1}}{d \eta}\right]+\frac{1}{\beta}\left[\frac{x_{1}-p}{k d}\right]^{p_{2}+p_{1}-p_{0}} } \\
& \left.\times f_{2} \frac{f_{1}}{d \eta}-\frac{1}{\beta}\left[\frac{x_{1}-p}{k d}\right]^{p_{2,1}-p_{0}} \frac{1}{\eta} \frac{d}{d \eta}\left(\eta f_{2,1}\right)\right\}=0 .
\end{aligned}
$$

From the self-similarity of the following scaled correlation, we obtain

$$
\frac{\overline{u_{2}^{\prime} u_{1}^{\prime}}}{{\overline{u_{1}^{2}}}^{2}}=2 p_{1}-p_{2,1}=0 \text {. }
$$

Self-similarity only occurs in two limits, the first being

$$
\frac{U}{U_{G}} \gg 1
$$

The additional velocity of the jet $U$ at the outlet of the nozzle is an order of magnitude higher than the velocity of the basic flow $U_{G}$ (Fig. 2). This assumption leads to two further relations and some simplifications of (55) and (56):

$$
\left.\begin{array}{r}
p_{0}+p_{1}-p_{2}=1 \\
p_{0}+2 p_{1}-p_{2,1}=1 \\
p_{0}+p_{1}=0 \\
2 p_{1}-p_{2,1}=0
\end{array}\right\} \Rightarrow p_{0}=1, p_{1}=-1, p_{2}=-1, p_{2,1}=-2 \text {, }
$$

$$
f_{1}+\eta \frac{d f_{1}}{d \eta}-\frac{1}{\beta} \frac{1}{\eta} \frac{d}{d \eta}\left(\eta f_{2}\right)=0
$$

and

$$
f_{1}^{2}+\eta f_{1} \frac{d f_{1}}{d \eta}-\frac{1}{\beta}\left[f_{2} \frac{d f_{1}}{d \eta}-\frac{1}{\eta} \frac{d}{d \eta}\left(\eta f_{2,1}\right)\right]=0 .
$$

From (60) after a partial integration, it follows that

$$
f_{2}=\beta\left(\eta f_{1}-\frac{1}{\eta} \int_{0}^{\eta} \xi f_{1}(\xi) d \xi\right) .
$$

The difference-quotient turbulence model written in terms of the self-similar variable $\eta$ is

$$
\begin{aligned}
\overline{u_{2}^{\prime} u_{1}^{\prime}} & =-b \frac{d b}{d x_{1}} \overline{u_{1}^{*}}\left(x_{1}, x_{2}\right) \frac{\overline{u_{1}^{*}}\left(x_{1}, 0\right)-\overline{u_{1}^{*}}\left(x_{1}, x_{2}\right)}{-x_{2}} \\
& =f_{2,1}=-\beta \frac{1}{\eta} f_{1}\left(1-f_{1}\right),
\end{aligned}
$$

because (see Fig. 2)

$$
x_{2_{\max }}=0, \overline{u_{1_{\min }}}=0, \overline{u_{1_{\max }}}=\overline{u_{1}}{ }^{*}\left(x_{1}, 0\right) .
$$

If we insert (62) and (63) into (61), the following form is obtained:

$$
\int_{0}^{\eta} \xi f_{1}(\xi) d \xi=1-2 f_{1}-\eta \frac{f_{1}^{2}}{f_{1}^{\prime}},
$$

where we have introduced the abbreviation

$$
\chi^{\prime}=\frac{d \chi}{d \eta}
$$

Taking the derivative of (65), and substituting an expression given by the same equation (65), leads to the highly nonlinear differential equation

$$
\eta f_{1}^{2} f_{1}^{\prime \prime}-2\left(f_{1}^{\prime}\right)^{3}-3 \eta f_{1}\left(f_{1}^{\prime}\right)^{2}-\left(f_{1}\right)^{2} f_{1}^{\prime}=0 \text {. }
$$

After substituting:

$$
f_{1}=\exp [g(\eta)]
$$

a somewhat simpler differential equation results:

$$
\eta g^{\prime \prime}-2\left(g^{\prime}\right)^{3}-2 \eta\left(g^{\prime}\right)^{2}-g^{\prime}=0 \text {. }
$$

The solution of this equation is

$$
g=-\frac{1}{2} \eta^{2}+\text { const . }
$$

We obtain a Gaussian function for the radial distribution of the dimensionless mean velocity in the main flow direction:

$$
f_{1}=\exp \left(-\frac{1}{2} \eta^{2}\right) .
$$

Experimental results are found in Refs. [16-19]. The analytical calculations in this section, obtained with the difference-quotient model, are compared with the measurements performed by Panchapakesan and Lumley [19]. In their paper they give an estimation of

$$
K_{u}=\frac{1}{2 \beta^{2}}=75.2 \Rightarrow \beta=0.082 \text {. }
$$

For all comparisons of calculations with measurements this value of the spreading parameter $\beta$ has been used. It corresponds to a spreading angle of the turbulent round 
jet of $9.4^{\circ}$. Figure 3 experimentally confirms the selfsimilarity of the investigated velocity profiles. Data points are given for different values of downstream distances $s / d$. They clearly show the scaling behavior which has been assumed in the theoretical treatment of this section.

Continuing, after an integration of Eq. (62), we obtain

$$
f_{2}=\beta\left\{\eta \exp \left(-\frac{1}{2} \eta^{2}\right)-\frac{1}{\eta}\left[1-\exp \left(-\frac{1}{2} \eta^{2}\right)\right]\right\} .
$$

Panchapakesan and Lumley have not measured the mean velocity in the radial direction $f_{2}$ independently. They have calculated this distribution taking a least-squares spline fit of the data set of $f_{1}$ and substituting it into the continuity equation. Therefore, a comparison of the experimental values of $f_{2}$ with results obtained by Eq. (73) would also show good agreement, but without being a further test of the model. In Fig. 4 it can be seen that the vertical mean velocity has negative values some distance from the axis; a flow occurs towards the jet axis. In fluid dynamics this phenomenon is well known and is named entrainment. Note that at its maximum $f_{2}$ only reaches $2 \%$ of the maximal value of $f_{1}$. By substituting (71) into (63) the tangential Reynolds stress can be derived, which is a linear combination of two exponential functions:

$$
f_{2,1}=-\beta \frac{1}{\eta}\left[\exp \left(-\frac{1}{2} \eta^{2}\right)-\exp \left(-\eta^{2}\right)\right] .
$$

As shown in Fig. 5, the calculated Reynolds shear stress is also in good agreement with experimental results.

For the mean velocity in the main flow direction-on the jet axis-the following equation can be derived (see Ref. [20]):

$$
{\overline{u_{1}}}^{*} \frac{d \bar{u}_{1}^{*}}{d x_{1}}-\frac{p_{1}}{x_{1}-p} \overline{u_{1}^{* 2}}=0
$$

whereas the exponent of the power law describing $\overline{u_{1}}$ * occurs as a coefficient:

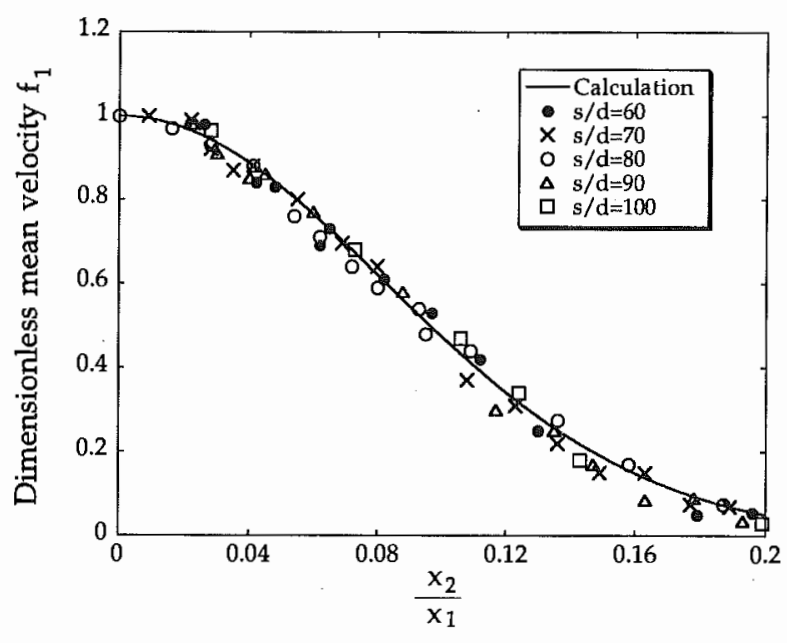

FIG. 3. Comparison of the calculated radial distribution of the mean velocity in the main flow direction with experimental results (from Ref. [19]).

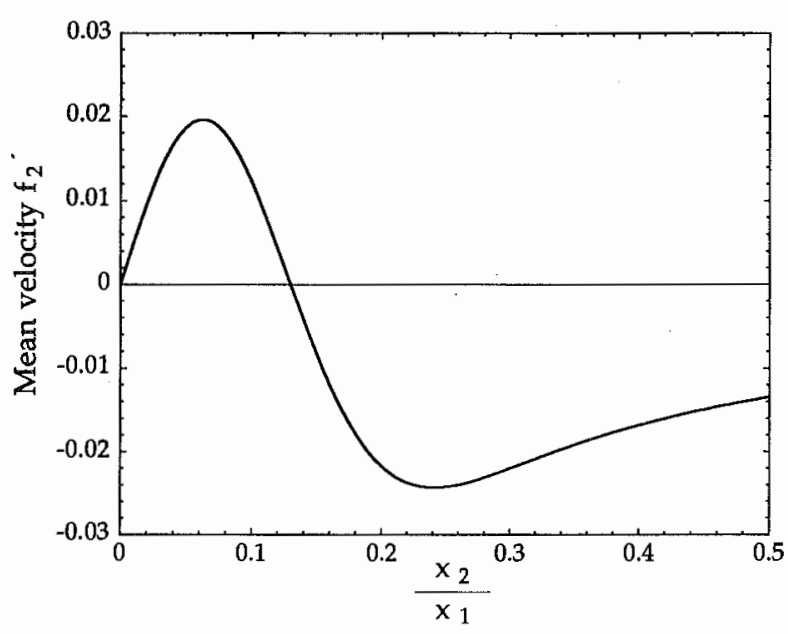

FIG. 4. The scaled radial distribution of the mean velocity perpendicular to the axis of the jet shows entrainment $\left(f_{2}<0\right)$.

$$
\begin{aligned}
p_{1}=-\left.2 \frac{\partial\left[\frac{\overline{u_{2}^{\prime} u_{1}^{\prime}} *\left(x_{1}, \frac{x_{2}}{x_{1}}\right)}{\overline{u_{1}^{*}\left(x_{1}, 0\right)^{2}}}\right]}{\partial\left(\frac{x_{2}}{x_{1}}\right)}\right|_{x_{2} / x_{1}=0} . \\
=\left.2 \frac{\partial f_{2,1}}{\partial\left(\frac{x_{2}}{x_{1}}\right)}\right|_{x_{2} / x_{1}=0} .
\end{aligned}
$$

A Taylor expansion of the Reynolds shear stress (74), predicted by our model, in the limit $x_{2} / x_{1} \rightarrow 0$, has the following form:

$$
f_{2,1}=-\frac{1}{2}\left(\frac{x_{2}}{x_{1}}\right)+O\left[\left(\frac{x_{2}}{x_{1}}\right)^{2}\right] .
$$

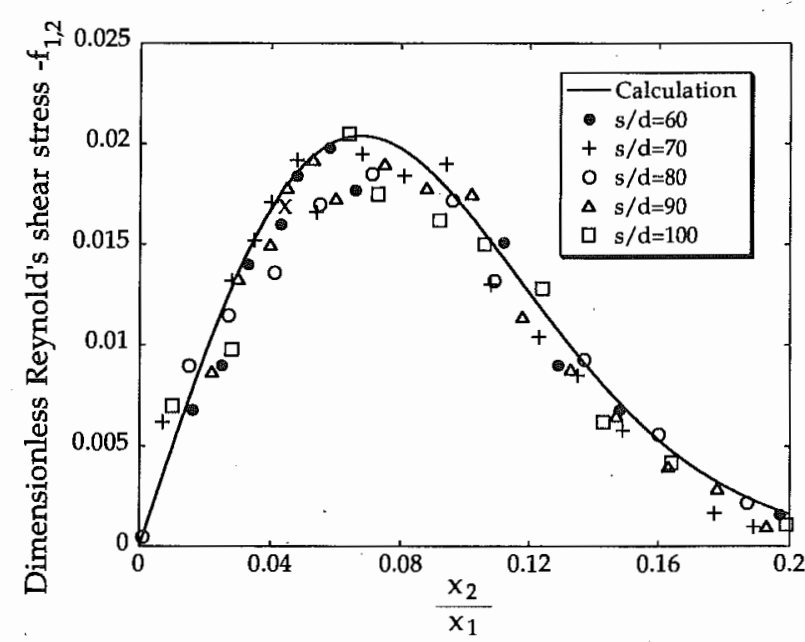

FIG. 5. The radial distribution of the Reynolds shear stress. Experimental data are from Ref. [19]. 
Substituting Eq. (77) into (76) yields $p_{1}=-1$, just as required by the similarity relations [compare with (59)]. Therefore, the exponent of the power law describing the behavior of $\bar{u}_{1}^{*}$-obtained with the difference-quotient model-is also consistent with the absolutely necessary requirement given by the conservation laws of mass and momentum.

\section{Turbulent round jet in a coflow with high velocity}

The second case showing self-similarity occurs if

$$
\frac{U}{U_{G}} \ll 1
$$

Because this condition is fulfilled in many combustion problems it is of great practical importance, especially in chemical engineering.

Equations (54) and (57) are still valid and together with (56) and (78) it follows that

$$
\left.\begin{array}{r}
p_{0}+p_{1}-p_{2}=1 \\
p_{0}+p_{1}-p_{2,1}=1 \\
2 p_{0}+p_{1}=0 \\
2 p_{1}-p_{2,1}=0
\end{array}\right\} \Longrightarrow p_{0}=\frac{1}{3}, p_{1}=-\frac{2}{3}, p_{2}=-\frac{4}{3}, p_{2,1}=-\frac{4}{3} \text {. }
$$

Now the continuity and momentum equation are

$$
\begin{aligned}
& 2 f_{1}+\eta \frac{d f_{1}}{d \eta}-\frac{3}{\beta} \frac{1}{\eta} \frac{d}{d \eta}\left(\eta f_{2}\right)=0 \\
& 2 f_{1}+\eta \frac{d f_{1}}{d \eta}+\frac{3}{\beta}\left(\frac{U}{U_{G}}\right) \frac{1}{\eta} \frac{d}{d \eta}\left(\eta f_{2,1}\right)=0 .
\end{aligned}
$$

The difference-quotient model again applies with no problems $\left(\chi_{2}=b\right)$ :

$$
\begin{aligned}
\overline{u_{2}^{\prime} u_{1}^{\prime}}= & b \frac{d b}{d x_{1}}\left[U_{G}+{\overline{u_{1}}}^{*}\left(x_{1}, x_{2}\right)\right] \\
& \times \frac{\left[U_{G}+{\overline{u_{1}}}^{*}\left(x_{1}, 0\right)\right]-\left[U_{G}+{\overline{u_{1}}}^{*}\left(x_{1}, x_{2}\right)\right]}{x_{2}} \\
\Longrightarrow & f_{2,1}=-\frac{\beta}{3}\left(\frac{U_{G}}{U}\right) \frac{1}{\eta}\left(1-f_{1}\right),
\end{aligned}
$$

after applying an approximation given by (78). Substituting (82) into (81) yields

$$
2 f_{1}+\eta \frac{d f_{1}}{d \eta}+\frac{1}{\eta} \frac{d f_{1}}{d \eta}=0
$$

When this equation is integrated, the inner derivative is present in the numerator. Taking also (80) and (81) into consideration, immediately some analytical solutions follow:

$$
\begin{aligned}
& f_{1}=\frac{1}{1+\eta^{2}}, f_{2}=\frac{\beta}{3} \frac{\eta}{1+\eta^{2}} \\
& f_{2,1}=-\frac{\beta}{3}\left[\frac{U_{G}}{U}\right] \frac{\eta}{1+\eta^{2}} .
\end{aligned}
$$
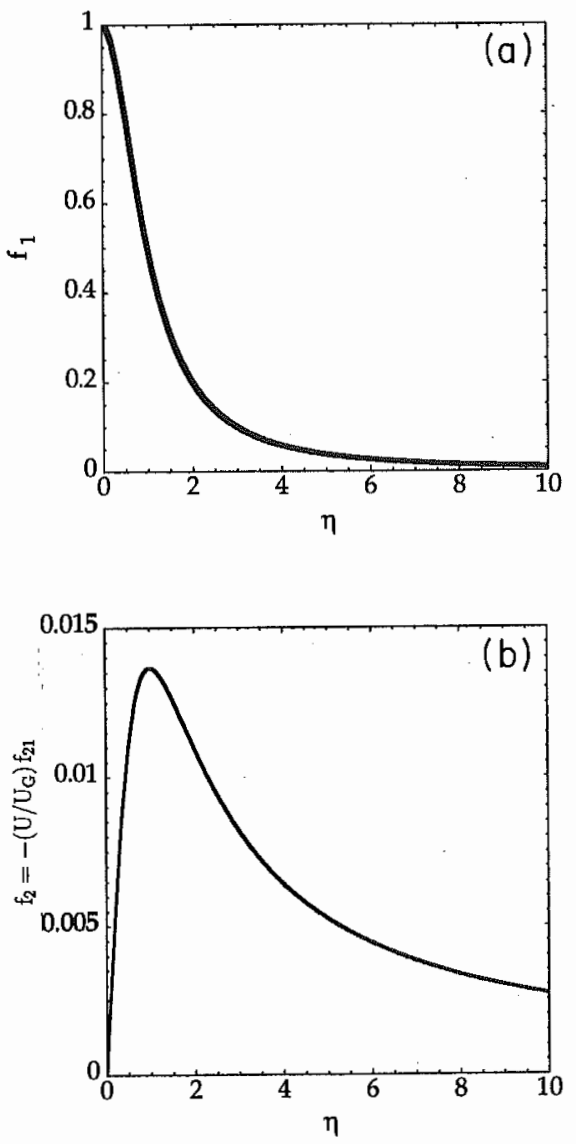

FIG. 6. Several mean properties of a round turbulent jet in a high-velocity surrounding flow with a low excess outlet velocity.

These functions are shown in Fig. 6. In combustion problems chemical processes are involved and very often the jets have rotating cores. That is the reason why no adequate data were found to compare the theoretical predictions with measurements. Nevertheless, the profiles are qualitatively absolutely correct. The mean velocity profile in the main flow direction $f_{1}$ shows a maximum at zero and vanishes continuously towards infinity. Because of symmetry requirements the mean vertical velocity $f_{2}$ and the Reynolds shear stress $f_{2,1}$ have to vanish at the origin $(\eta=0)$, which is indeed the case. As desired, they both also approach zero when $\eta$ tends to infinity. Interesting is that in this case, the function $f_{2}$ does not change sign. Therefore, a further prediction of the model is that a turbulent round jet with a low excess outlet velocity in a uniform high-velocity surrounding does not show the entrainment phenomenon.

\section{CONCLUSIONS}

The aim of this paper was not to give a contribution to one of the most sophisticated modern methods of turbulence calculation and/or modeling, e.g., transport equation models or large eddy simulation. The intention was to recall some older approaches from Prandtl and to connect them with this recently derived differencequotient model: a turbulence model with no empirical constants. This theory contains derivatives of infinite or- 


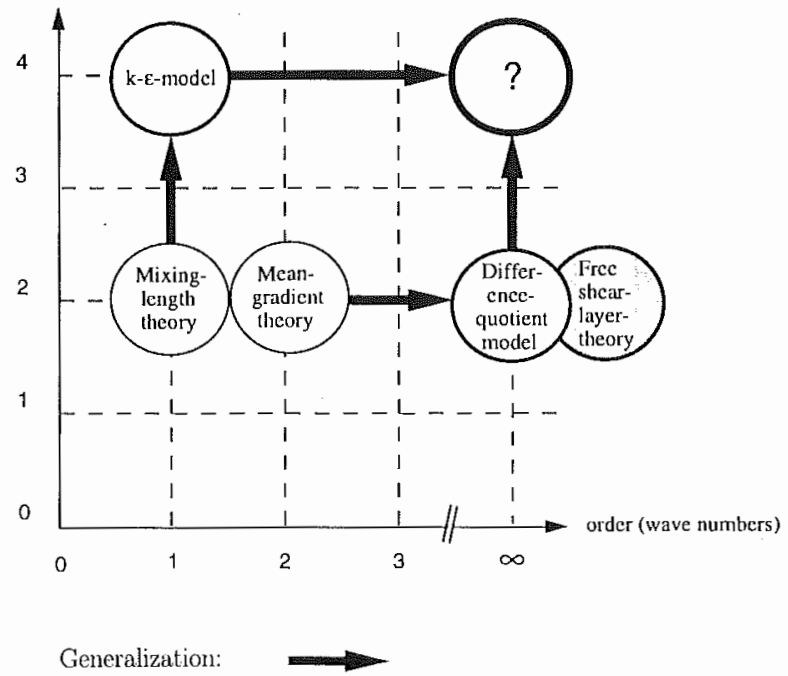

Ordinatc valucs:

$\begin{array}{ll}\text { 1: } P, & \text { P: Production by shear stress } \\ \text { 2: P+V, } & \text { V: Viscous dissipation } \\ \text { 3: } P+V+C, & \text { C: Convective transport } \\ \text { 4: } P+V+C+D, & \text { D: Diffusive transport }\end{array}$

FIG. 7. Some turbulence models and their domains of application.

der. Applying Fourier transformation techniques, we find that the model corresponds to an infinitely large number of wavelengths or eddy sizes. Figure 7 shows the number of energy terms in the energy equation of turbulent flows - for simplicity, e.g., pressure and buoyancy effects are included in the difusive term [21] - relative to the number of wave numbers occurring in a model. Only such an infinite-order theory is compatible with energycascade considerations, such as, for example, those carried out by Kolmogorov and Obukhov in 1941. On the other hand, the model is restricted to systems with a turbulence in local mechanical equilibrium, which is characterized by production and dissipation of turbulent kinetic energy only $(\infty, 2)$. More recent approaches with gradient laws, such as the standard $k-\epsilon$ model, can be used to describe more complex flow situations $(1,4)$; e.g., see [22]. From our discussion it becomes clear that such models are only first-order approaches to any given possible turbulent flow processes. On the other hand, we know that the difference-quotient model at least describes simple flows, such as turbulent shear flows, correctly. Despite this subtle difference, what we further must attempt to invent is a model of type $(\infty, 4)$, containing all the advantages of the model $(1,4)$ and the approach $(\infty, 2)$. Recent approaches, such as the renormalization-group $k-\epsilon \bmod -$ el, already tend in this direction.

In my opinion the importance of large-scale space and velocity differences, as they here occur in the description of second-order correlations-with no dependence on the velocity profiles in some open intervals between distinct points - could be a basic feature of (fully developed) turbulence. Hopefully, this phenomenon will be explained in terms of a statistical theory of turbulence one day.

\section{ACKNOWLEDGMENTS}

I wish to thank J. L. Olsen and R. Künzli for helpful advice and discussions. The author is indebted to $\mathbf{N}$. Hopkirk for her comments on a first draft of this paper. Furthermore, I thank F. Busse for also suggesting the addition of Sec. $V$ (the performance of the model) and for making remarks relevant to it.
[1] R. J. Donnelly, Phys. Today 44 (11), 32 (1991).

[2] J. O. Hinze, Turbulence, 2nd ed. (McGraw-Hill, New York, 1975), Chap. 6.

[3] G. Speziale, Phys. Fluids 22, 1033 (1979).

[4] J. Boussinesq, Mém. Prés. Div. Savant Acad. Sci. Paris 23, 46 (1872).

[5] H. Tennekes and J. L. Lumley, A First Course in Turbulence (MIT Press, Cambridge, MA, 1972), Chap. 2.

[6] S. Corrsin, Adv. Geophys. 18A, 25 (1974).

[7] P. S. Bernard and R. A. Handler, J. Fluid Mech. 220, 99 (1990).

[8] L. Prandtl, Z. Angew, Math. Mech. 5, 136 (1925).

[9] M. M. Stanišić, The Mathematical Theory of Turbulence, 2nd ed. (Springer-Verlag, Berlin, 1988), Chap. I/3.

[10] J. R. Holton, An Introduction to Dynamic Meteorology, International Geophysics Series Vol. 23 (Academic, New York, 1979), Chap. 5.

[11] L. Prandtl, Z. Angew. Math. Mech. 22, 241 (1942).
[12] H. Görtler, Z. Angew. Math. Mech. 22, 244 (1942).

[13] Th. von Karman, Nachr. Ges. Wiss. Göttingen, Math. Phys. K1. 5, 58 (1930).

[14] P. W. Egolf, Helv. Phys. Acta 64, 944 (1991).

[15] P. J. Strykowski and D. L. Niccum, J. Fluid Mech. 227, 309 (1991).

[16] I. Wygnanski and H. Fiedler, J. Fluid Mech. 38/3, 577 (1969).

[17] W. Rodi (unpublished).

[18] S. P. Capp, H. J. Hussein, and W. K. George, Turbulence Research Laboratory, University at Buffalo Technical Report No. 123, 1990 (unpublished).

[19] N. R. Panchapakesan and J. L. Lumley, J. Fluid Mech. 246, 197 (1993).

[20] P. W. Egolf, Gesund. Ing. 4, 198 (1992).

[21] J. C. Rotta, Turbulente Strömungen (Teubner-Verlag, Stuttgart, 1972), Chap. 3.

[22] B. E. Launder, Appl. Sci. Res. 48, 247 (1991). 